\title{
Principal component analysis of the modified clinical test of sensory interaction in healthy adult humans
}

\author{
OSEIKHUEMEN DAVIS OJIE, REZA SAATCHI \\ Industry \& Innovation Research Institute (I2Ri), \\ Faculty of Science, Technology and Arts, Sheffield Hallam University, \\ Sheffield, UNITED KINGDOM
}

\begin{abstract}
A number of mechanisms and sensory systems in humans are associated with the maintenance of balance. Diagnosis and monitoring of balance dysfunctions could be assisted by exploring deviations of data recorded from patients with comparative or reference data from healthy individuals. To this effect, principal component analysis (PCA) was applied to accelerometry obtained time domain balance data. The data were recorded from 21 healthy adults ( 10 males and 11 females, mean age 24.5 years, standard deviation 4.0 years, mean height $173.6 \mathrm{~cm}$, standard deviation $6.8 \mathrm{~cm}$, and mean weight $72.7 \mathrm{~kg}$, standard deviation $9.9 \mathrm{~kg}$ ) in the medio-lateral (ML) and anterior- posterior (AP) directions. The subjects performed tasks specified in the modified clinical test of sensory interaction on balance (mCTSIB) while an accelerometry device was attached at their lower back, in the position of the iliac crest. Eighteen-time domain measures that quantified body's displacement, velocity and acceleration were obtained and processed using PCA. Based on the observations from PCA, further investigations were carried out on the root mean square (RMS) velocity using the Bland and Altman plots and other statistical related analysis. It was observed that the anterior and posterior directions were more sensitive to the absence or presence of balance sensory (visual, somatosensory and vestibular) inputs as compared to the mediolateral (ML) direction. A greater coherence in sway information was observed in the somatosensory system as compared to the visual and vestibular systems. There was more stability in the interaction between the somatosensory and the vestibular systems as compared to that of the visual and vestibular systems. The results obtained could be helpful to clinicians in balance related analysis and diagnosis.
\end{abstract}

Keywords: sensory interaction, balance dysfunctions, mCTSIB, Accelerometry

Received: May 18, 2020. Revised: October 27, 2020. Accepted: November 30, 2020. Published: December 28, 2020.

\section{Introduction}

The ability to maintain balance is crucial in performing daily activities safely and effectively. Balance in humans is primarily based on integration of information from the visual, somatosensory and vestibular systems [1] [2]. The understanding of these systems is imperative in determining the manner they contribute to balance. The modified clinical test of sensory interaction on balance (mCTSIB) allows the relationships between the relevant sensory systems' contributions to balance to be examined. It was derived from the clinical test of sensory interaction with balance (CTSIB) [3] that has six conditions: (i) standing on a firm surface with eyes open, (ii) standing on a firm surface with eyes closed, (iii) standing on a firm surface with a visual conflict dome, (iv) standing on a compliant surface with eyes open, (v) standing on a compliant surface with eyes closed and (vi) standing with a visual conflict dome on a compliant surface [4]. The mCTSIB examines the subject under four balance-related sensory conditions. In condition one, the subject stands on a firm surface with eyes open. In this condition all the three balance-related sensory systems (i.e. visual, somatosensory and vestibular) contribute to maintenance of balance unhindered. The second condition is similar to the first but the visual input is excluded by the eyes being closed. The third condition is as the first condition but the input from the somatosensory system is affected, e.g. by the person standing on a flexible surface such as thick foam. Finally, the forth condition involves the subject standing on a flexible surface with eyes closed, i.e. affecting both the visual and somatosensory systems.

A number of studies have used the mCTSIB to investigate balance dysfunctions. The 
investigation of postural sway and its relation to stereopsis function in patients with rapid eye movement (REM) sleep behaviour disorder (RBD) have been reported [5]. Using accelerometers and with the eyes open and closed conditions, they found that a subtle sign of postural sway instability existed in patients with idiopathic RBD, most especially in subjects with abnormal stereopsis [5]. Postural sway measurements obtained using an accelerometer were compared to those obtained using a force platform in patients with untreated Parkinson's disease (PD) in scenarios of the eyes open and closed conditions of the MCTSIB and accelerometer based sway metrics [6]. They concluded that accelerometer-based sway metrics could be used as objective measures of postural instability in untreated PD patients [6]. In some studies, the analysis had been based on the comparison of balance parameters between patients and healthy individuals. Thus, there is a need to investigate balance patterns in healthy subjects to allow possible deviations from the expected measures to be better interpreted in patients. Principal component analysis (PCA) is a statistical technique used mainly for data dimensionality reduction and detecting differentiating patterns [7][8]. The use of PCA in finding distinctive features in balance and gait related analysis has been reported in a number of studies. Acceleration and velocity of the tibial translation information obtained using electromagnetic sensors were analysed using PCA [9]. The study included 127 pivot shift examinations by orthopaedic surgeons on 70 subjects presenting various degrees of knee joint instability. They determined the features that explained the observed variabilities [9]. PCA of postural movement data in fifty healthy adults involved in 80 seconds tandem stance indicated that age altered the postural control system in specific task relevant components [10]. The results obtained by applying PCA to the accelerometer obtained data of 43 young and 100 older subjects showed that the ratio of the mediolateral and vertical derivative of acceleration was a distinct gait construct [11]. In this study, we investigated the patterns of postural sway in healthy adults by analysing accelerometry data obtained during mCTSIB. Significant deviations from the postural sway patterns observed in healthy subjects may be indicative of possible balance dysfunctions. To carry out the analysis, PCA was employed owing to its effectiveness in kinematic investigations. We specifically explored the distinctive characteristics between the medial-lateral (ML) and anterior-posterior (AP) directions in relation to balance. The hypotheses investigated were:

- The AP direction may be more sensitive to postural sway as compared to the ML direction due to a larger area of the base of support and the observance of the concepts of inverted pendulum.

- No difference in sway between the interaction of the somatosensory and visual systems with the vestibular system. This hypothesis explored the uniformity and performance of the somatosensory and visual systems in relation to balance.

In the following sections, the accelerometry for sway path analysis and PCA are briefly described then the methodology and results are discussed.

\section{Accelerometry based sway analysis}

Human balance analysis in a standstill position can be described using the inverted pendulum model that has a centre of mass (COM) above its base of support [12]. Accelerometry requires the use one or more accelerometers to record and interpret motion. It captures the motion of the COM position and other areas of the human body. Accelerometry is gaining applications in a number of medical monitoring and diagnostic fields due to its cost effectiveness, portability and flexibility in implementation. A model to project COM sway to the ground surface has been developed [13] and extended based on the inverted pendulum in [14]. In this model, the sway at the COM position is projected downwards to the ground surface. The projection is shown in Figure 1. In the Figure, $R$ represents the resultant acceleration (unit $\mathrm{cm} / \mathrm{s}^{2}$ ) obtained from the orthogonal coordinates of the accelerations $a_{x}, a_{y}$ and $a_{z}$ of all three accelerometer axes (i.e. $x, y$ and $z$ ); $\alpha, \beta$, and $\gamma$ are angles (unit degrees) made by each coordinate to the resultant obtained from directional cosines, i.e. $\cos (\alpha), \cos (\beta)$, and $\cos (\gamma) ; d_{x}$ and $d_{y}$ (unit $\mathrm{cm}$ ) are the respective projected ground displacements in the mediallateral (ML) and anterior-posterior (AP) directions from the origin; $L$ represents the fixed position of the COM of the subject and $H$ is the inclined height of the COM above the ground surface 


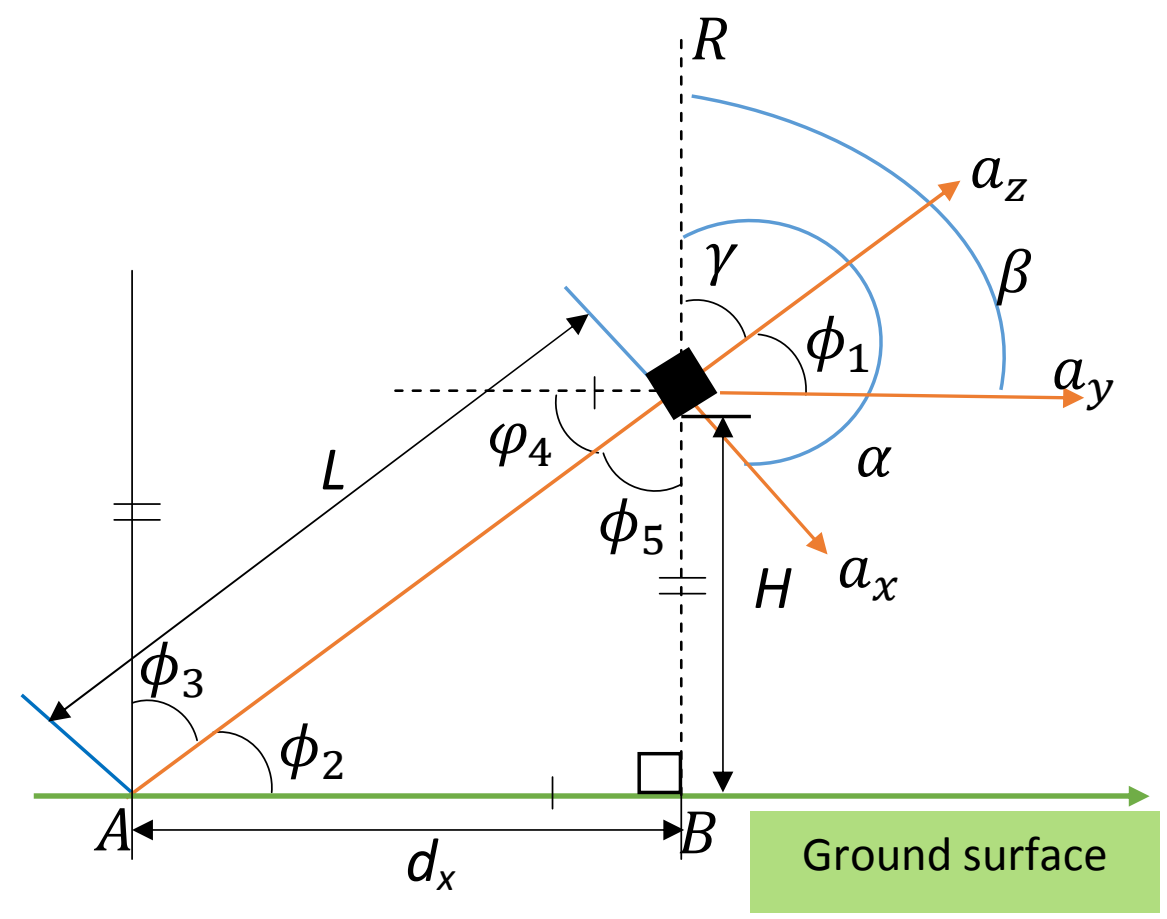

Figure 1. Inverted pendulum ground projection of COM sway (Ojie et al., 2020).

The formulae to obtain the above measures are given in equations 1 to 3 , where $\varphi_{1}, \varphi_{2}, \varphi_{3}, \varphi_{4}$; and $\varphi_{5}$; are angles used for mathematical representations, $A$ is the point of origin and point $B$ is deviation from the origin.

$$
\begin{aligned}
& \sqrt{a_{x}^{2}+a_{y}^{2}+a_{z}^{2}} \quad \cos (\alpha)=\frac{a_{x}}{A}, \cos (\beta) \\
& =\frac{a_{y}}{A}, \cos (\gamma) \\
& =\frac{a_{z}}{A} \\
& \quad d_{y}=-L \cos (\beta) \\
& \quad d_{x}=-L \cos (\gamma)
\end{aligned}
$$

\section{Brief introduction to principal component analysis}

Principal component analysis (PCA) is a technique mainly used for data reduction while preserving the majority of the information in the original data. Consider a matrix $(\boldsymbol{D})$ of order $m \times n$ containing the data set with $m$ observations (rows) and $n$ variables (columns). In this study, $m$ is the number of subjects and $n$ is the number of time domain variables.

$$
\boldsymbol{D}_{m \times n}=\left(\begin{array}{ccc}
d_{1,1} & \ldots & d_{1, n} \\
\vdots & \vdots & \vdots \\
d_{m, 1} & \ldots & d_{m, n}
\end{array}\right)
$$

The eigenvalues and vectors of the correlation or cofvariance matrix obtained by eigenvalue decomposition represent the magnitudes of the variance and their principal components respectively. The principal components are ranked in order of the magnitudes of their eigenvalues, i.e. the eigenvector with the maximum eigenvalue is referred to as the first principal component and so on. Prior to its application on a data set, the variables are standardised in order to prevent variables with relatively larger magnitudes from suppressing those with lower magnitudes. Some common standardization techniques include: the minimum-maximum, and the z-score. Prior standardisation is not required when correlation matrix is used [15]. The majority of the variation in PCA is usually explained by selecting the first few components with the largest eigenvalues. The components that correspond to smaller eigenvalues can be excluded as they represent a relatively small proportion of variance and may 
carry relatively little relevant information. In this way, PCA ensures a reduction in the dimensionality of the dataset. The number of retained principal components can be determined by eigenvalues greater than 1 and/or by using the scree plot analysis [16] [17]. The matrix representing the correlation of the components with the variables is referred to as the component matrix. It is often necessary that the component matrix be rotated to obtain a representative structure, i.e. a pattern where only one variable loads or correlates highly unto a component. There are many different types of rotation that can be carried out after the initial extraction of the components. In this study, varimax rotation is employed. Varimax rotation is an orthogonal rotation that maximises the sum of variances of the squared coefficient within each eigenvector [18]. Usually, inference is based on the relationship of the correlations of the variables with the components in what is referred to as factor loadings. Factor loadings are the coefficients of the correlations between the components and the variables. Variables with closely related correlation coefficients (loadings) have a similar relationship, i.e. their standardised values are closely related. Variables with relatively high correlation coefficients are referred to as representatives of the principal components [19]. In other to consider a variable to correlate with a component, factor loadings greater than 0.4 are considered to have reached the basic minimal significance level [20]. In this study, the threshold for a variable to load was 0.4 .

\section{Methodology}

\subsection{Accelerometry device used for data recordings}

An accelerometry device was developed to carry out the data recordings. The device, shown in Figure 2, consisted of transmitter and receiver units. The transmitter unit measured the body's sway. It consisted of an MPU6050 inertial measurement unit (IMU), an Arduino Nano and an nrf24L01 wireless transceiver. The receiver unit interfaced with a laptop computer through a USB connection and consisted of an nrf24L01 wireless transceiver and an Arduino Uno. The transmitter and receiver units were powered by a rechargeable battery and the laptop's USB connection
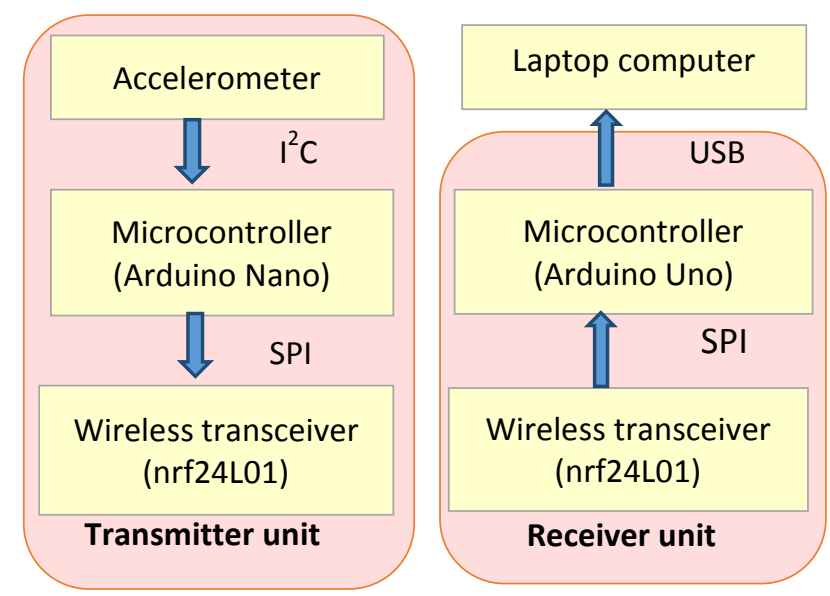

Figure 2. Accelerometry device devised for data recordings, consisting of transmitter and receiver units 
The Arduino Nano in the transmitter unit was programmed to communicate with the inertia sensor (an accelerometer) and to transmit the accelerometry information using its wireless transceiver to the receiver unit's wireless transceiver. The Arduino software was used for the programming of the microcontrollers. Furthermore, the Arduino Uno in the receiver unit communicated the received accelerometry information to the laptop computer using a USB interface connector, where the data were stored on its hard disk using the Processing Language based software. The communication protocols were: the Serial Peripheral Interface (SPI) that allowed communication between the microcontroller and the wireless transceivers, $\mathrm{I}^{2} \mathrm{C}$ that allowed communication between the inertial sensor and the microcontroller. The full scale range of the accelerometer was set to $\pm 2 g$ ( $g$ represents gravity, value about $9.81 \mathrm{~m} / \mathrm{s}^{2}$ ).

\subsection{Details of the subjects included in the study}

Twenty-one healthy adult subjects (10 males and 11 females), mean age 24.5 years and standard deviation 4.0 years, mean height $173.6 \mathrm{~cm}$ and standard deviation $6.8 \mathrm{~cm}$, and mean weight 72.7 $\mathrm{kg}$ and standard deviation $9.9 \mathrm{~kg}$ with no balance dysfunction participated in the study. The subjects declared not to have ingested anything (food, drinks, medication etc.) that could have affected their balance ability, forty-eight hours prior to the recordings. Corrective lenses or glasses were worn by those who used them.

\subsection{Data recording procedure}

Ethical approval to carry out the study was obtained from the university's ethics committee. The transmitting unit part of the device was worn by the subject as it was integrated into a belt as shown in Figure 3.



Figure 3. The transmitter unit worn by of the subjects. The subject has stood on a soft sponge pad as part of carrying out mCTSIB's conditions 3 and 4 tasks. 
The belt was arranged so that the transmitter unit was situated at the iliac crest of the lower back region. The subject stood with shoes off and feet around $30 \mathrm{~cm}$ apart. The subject was instructed to look to a point on the wall at a distance of about $1 \mathrm{~m}$, at eyes' level while performing the tasks associated with the conditions of the mCTSIB. The data recording for each test of mCTSIB was 30 seconds, during which the subject was relaxed. A resting time interval was provided in between the four mCTSIB tests. For mCTSIB conditions 3 and 4 , the subject had to stand on a flexible surface. The flexible surface used was a soft sponge pad, thickness $10 \mathrm{~cm}$, length and width $50 \mathrm{~cm}$. The data were transmitted wirelessly to a laptop computer and stored in its hard disk. The accelerometry data recording sample rate was 60 samples per seconds. The recorded data consisted of the numbers representing acceleration in the $x, y$ and $z$ directions of the accelerometer's axes contained in 3 columns. Each column contained 1800 sample recording (i.e. 30 seconds recording at 60 samples per second).

\subsection{Data processing and analysis}

The recorded accelerometry data were lowpass filtered using a forth order Butterworth filter with cut-off frequency of $4 \mathrm{~Hz}$. The filtering was performed to remove unwanted frequency components that could have obscured the analysis. They were then converted into unit $g$ by dividing them with the accelerometer's sensitivity scale factor (16384 least significant $\mathrm{bit} / \mathrm{g}$ ) for a full-scale range of $\pm 2 \mathrm{~g}$. The resulting axial accelerations $\left(a_{x}, a_{y}\right.$ and $\left.a_{z}\right)$ were converted into directional cosines (i.e. $\cos (\alpha)$ and $\cos (\beta))$ using equation 2 . The respective body sway displacements on the ground surface from the origin in the $x$ and $y$ axes (i.e. $d_{x}$ and $d_{y}$ ) were calculated using equation 3. Furthermore, the time domain measures in the ML and AP directions were obtained from these measures: i.e. associated displacements, velocities, and accelerations [21]. The features in the ML and AP directions as defined by equations 4 to 8 included: the ranges of the displacements $\left(\right.$ Range $\left._{z}\right)$, velocities $\left(\right.$ RangeV $\left._{z}\right)$ and accelerations $\left(\right.$ Range $\left._{z}\right)$, the averages of the displacements $\left(D_{z_{a v}}\right)$, velocities $\left(V_{z_{a v}}\right)$ and accelerations $\left(A_{z_{a v}}\right)$, and the root mean square (RMS) of the displacements $\left(D_{z_{R M S}}\right)$, velocities $\left(V_{Z_{R M S}}\right)$ and accelerations $\left(A_{Z_{R M S}}\right)$, where the subscript $z$ can either be ML or AP, depending on the direction under consideration. Velocity is the first derivative of displacement and acceleration is its second derivative with respect to time $(t)$ in seconds. The sampling interval $T=\frac{1}{f_{s}}$, where $f_{s}$ is the sampling frequency (i.e. 60 samples per second). To remove the bias introduced from the inclination of the device on the subject's body, the first value of the displacements was subtracted from the subsequent readings. Therefore, the measurements were representative of changes with reference to the first reading. The units of displacement, velocity and acceleration are $\mathrm{cm}, \mathrm{cm} / \mathrm{s}, \mathrm{cm} / \mathrm{s}^{2}$ respectively.

$$
\begin{array}{r}
D_{z_{n}}=d_{z_{n}}-d_{z_{1}}, V_{z_{n}}= \\
\frac{D_{z_{n}}-D_{z_{n-1}}}{T}, A_{z_{n}}=\frac{V_{z_{n}}-V_{z_{n-1}}}{T} \\
\text { (4) } \\
D_{z_{a v}}=\frac{1}{N} \sum_{n=1}^{N}\left|D_{z_{n}}\right|, V_{z_{a v}}= \\
\frac{1}{N} \sum_{n=1}^{N}\left|V_{z_{n}}\right|, A_{z_{a v}}=\frac{1}{N} \sum_{n=1}^{N}\left|A_{z_{n}}\right|
\end{array}
$$

$$
\text { Range } D_{z}=\mid \max \left(D_{z}\right)-
$$
$\min \left(D_{z}\right) \mid$

$$
\begin{aligned}
\operatorname{RangeV}_{z} & =\left|\max \left(V_{z}\right)-\min \left(V_{z}\right)\right|, \\
\text { Range }_{z} & =\left|\max \left(A_{z}\right)-\min \left(A_{z}\right)\right|
\end{aligned}
$$

$$
\begin{gathered}
D_{z_{R M S}}=\sqrt{\frac{1}{N} \sum_{n=1}^{N}\left(D_{z_{n}}\right)^{2}}, V_{z_{R M S}}= \\
\sqrt{\frac{1}{N} \sum_{n=1}^{N}\left(V_{z_{n}}\right)^{2}}, A_{z_{R M S}}=\sqrt{\frac{1}{N} \sum_{n=1}^{N}\left(A_{z_{n}}\right)^{2}}
\end{gathered}
$$

(8)

PCA was applied collectively on these features for each of the four conditions of mCTSIB. By applying PCA, the underlying structural relationships between the conditions were examined based on the similarities of their respective correlated matrices. Condition one of the mCTSIB was used as reference since it adapted information from all balance related sensory systems. Further investigations were carried out using the Bland and Altman plot, and other statistical techniques. The Bland and Altman plot is a method used to analyse the relationship between two variables in terms of their degree of similarity and agreement [22]. The acronyms used for the conditions are: condition one, eyes open standing on the ground surface (GEO), condition two, eyes closed 
standing on the ground surface (GEC), condition three, eyes open standing on foam (a soft sponge pad) surface (FEO) and condition four, and eyes closed standing on the foam surface (FEC). In this study, analysis was based on the assumption of a linear relationship between the balance related sensory systems. However, the interactions of these systems may not necessarily be linear in nature. Test to determine whether the data were from a normal distribution was carried out using the Shapiro-Wilk test. Based on this investigation, test for homogeneity of variance was carried out using the mean based Levene's test (i.e. if the data conformed to normality) or the median based Levene's test (i.e. if the data did not conform to normality). If the data met the condition of normality and homogeneity, oneway analysis of variance (ANOVA) was used to explore significant differences; otherwise Friedman test was used. When Friedman test was used, post hoc analysis was conducted based on
Wilcoxon Signed rank test (i.e. if the condition of symmetric was meant) otherwise the Sign test was used.

\section{Results and Discussion}

The displacement, velocity and acceleration plots for the four conditions of the mCTSIB for one of the subjects are shown in Figure 4. Visually, the displacement $(\mathrm{cm})$, velocity $(\mathrm{cm} / \mathrm{s})$ and acceleration $\left(\mathrm{cm} / \mathrm{s}^{2}\right)$ for mCTSIB's conditions one and two showed more sway in the ML direction as compared to the AP direction. In contrast to conditions one and two, although the displacement for conditions three and four showed a similar pattern, i.e. more sway occurred in the ML direction, the velocity and acceleration showed that the sway is more towards the AP direction. However, the displacement plot may not be an accurate indicator of sway as it is a change of position vectors, i.e. changes with respect to the origin.

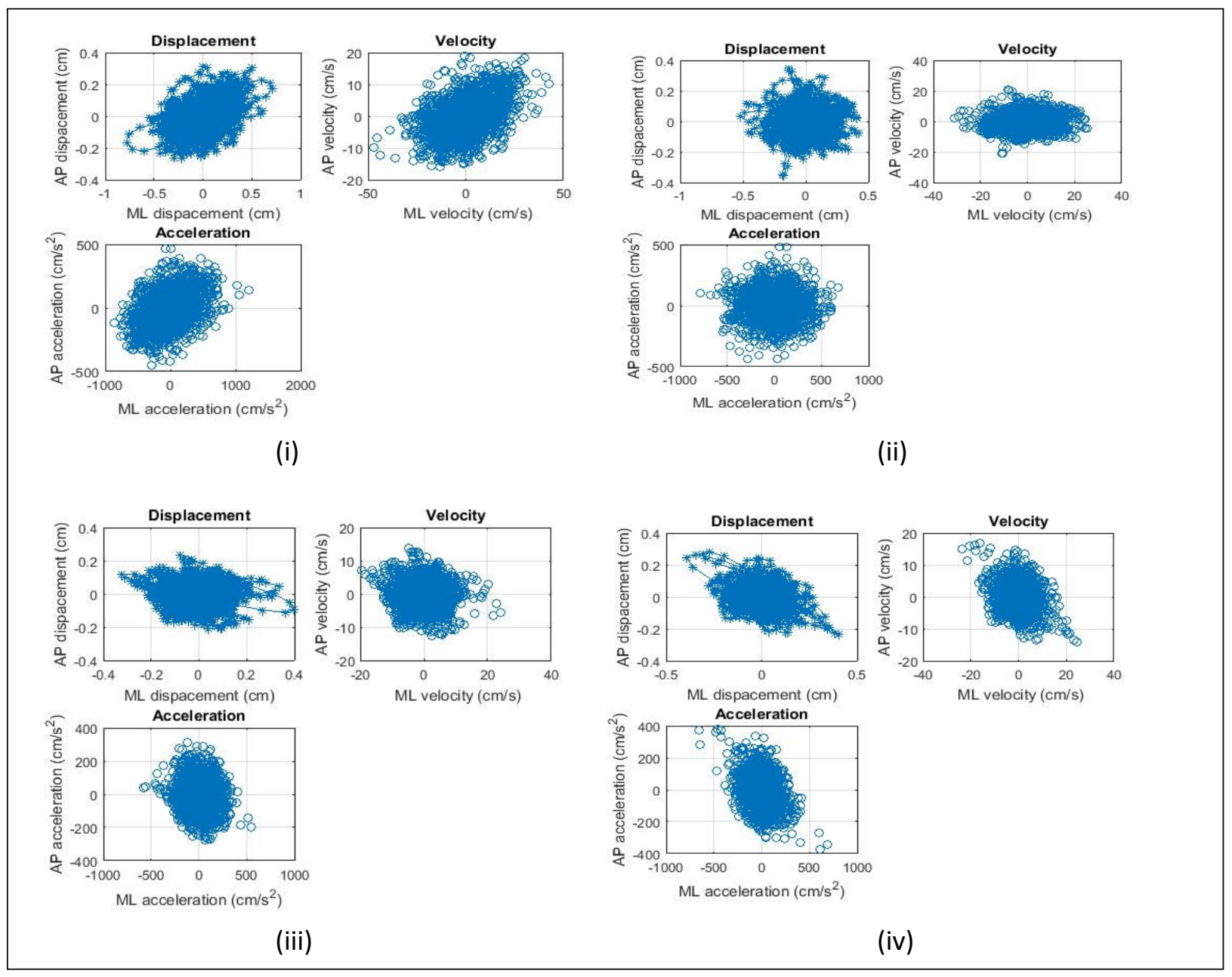

Figure 4. Representation of the displacement, velocity and acceleration of a subject for the four conditions ((i) - (iv)) of the mCTSIB. 
The scree plots obtained from the data for the four conditions of the MCTSIB are shown in Figures 5a - 5d. The components with corresponding eigenvalues above the knee of the scree plot were extracted. Three components were extracted for all the conditions and used for comparison purposes.
The variances represented by each component (PC1, PC2 and PC3) were: $67.7 \%, 14.1 \%$, and $9.1 \%$ for condition one, $65.9 \%, 14.3 \%$, and $9.2 \%$ for condition two, $60.9 \%, 18.3 \%$, and 9 . $4 \%$ for condition 3 , and $69.1 \%, 11.4 \%$ and $10.4 \%$ for condition 4 respectively.

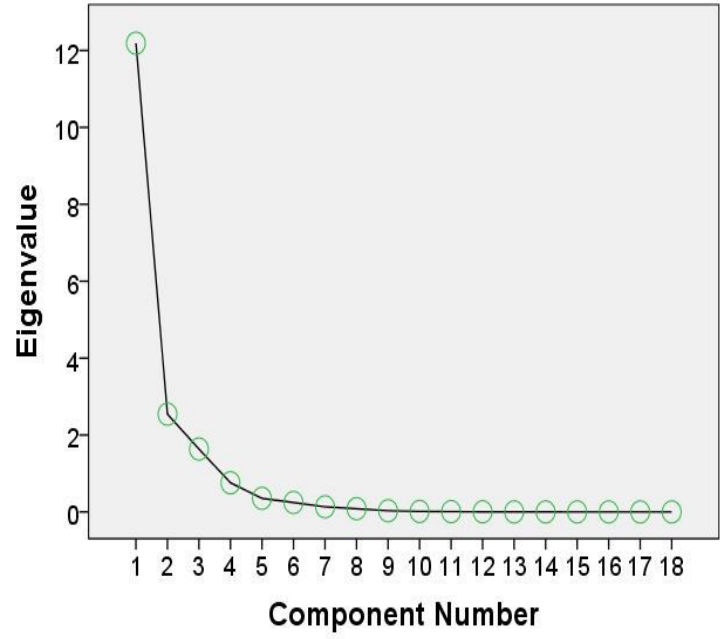

(a)

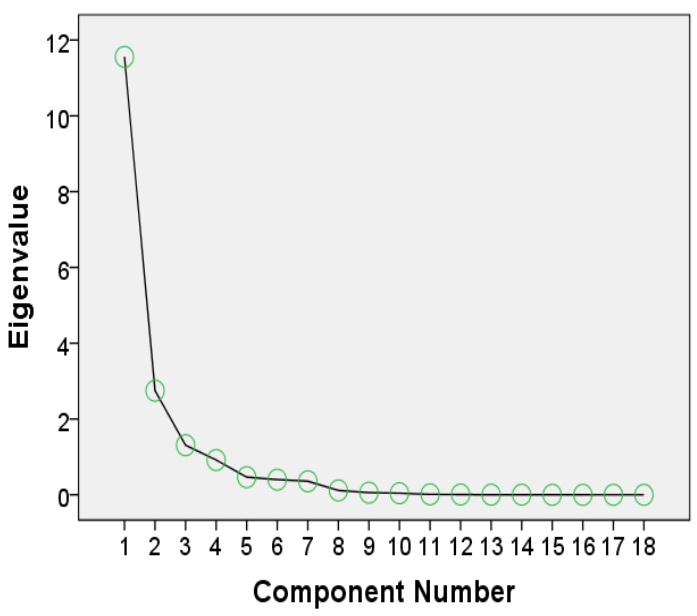

(c)

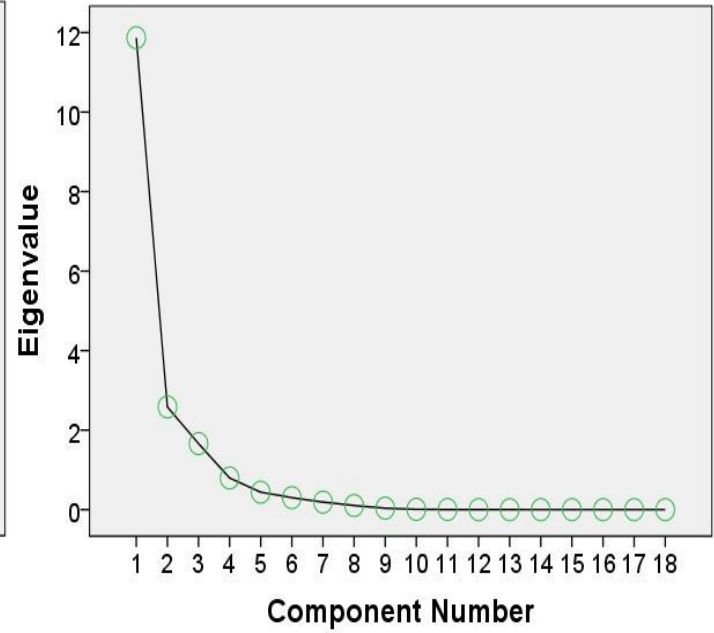

(b)

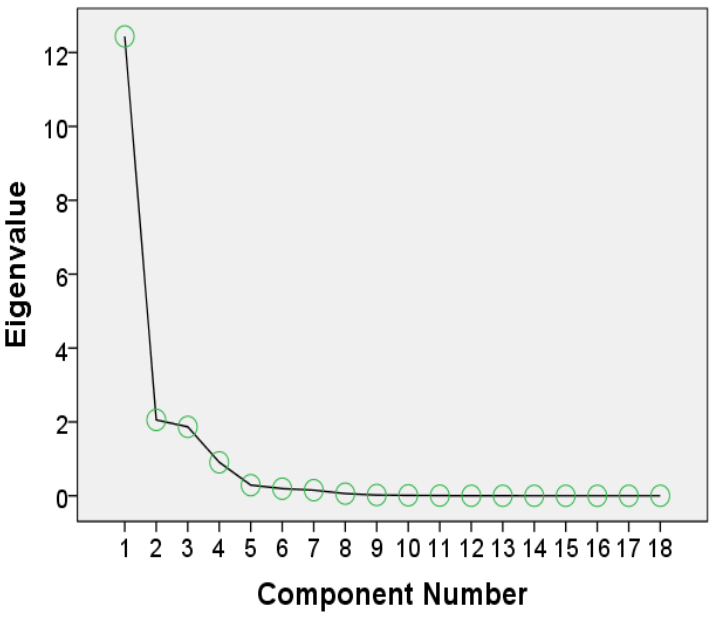

(d)

Figure 5. The scree plots of the 18 principal components: (a) condition one, (b) condition two, (c) condition three and (d) condition four of mCTSIB. 
5.1. Comparison between conditions one (GEO) and two (GEC)
The rotated component matrices for mCTSIB's conditions one and two are shown in Table 1.

Table 1. Rotated component matrix for conditions one and two.

\begin{tabular}{|c|c|c|c|c|c|c|c|}
\hline \multicolumn{8}{|c|}{$\mathrm{mCTSIB}$} \\
\hline \multirow[b]{2}{*}{ Number } & \multirow[b]{2}{*}{ Variables } & \multicolumn{3}{|c|}{ Condition 1} & \multicolumn{3}{|c|}{ Condition 2} \\
\hline & & PC1 & PC2 & PC3 & PC1 & PC2 & PC3 \\
\hline 1 & RangeD $_{\mathrm{ML}}$ & 0.780 & 0.503 & -0.064 & 0.661 & 0.661 & 0.097 \\
\hline 2 & RangeD $_{\mathrm{AP}}$ & -0.114 & -0.081 & 0.857 & -0.102 & 0.794 & 0.327 \\
\hline 3 & RangeV $_{\mathrm{ML}}$ & 0.843 & 0.399 & -0.222 & 0.700 & 0.632 & 0.174 \\
\hline 4 & RangeV $_{\mathrm{AP}}$ & 0.175 & 0.934 & -0.096 & 0.241 & 0.892 & 0.240 \\
\hline 5 & Range $A_{M L}$ & 0.774 & 0.541 & -0.199 & 0.701 & 0.614 & 0.180 \\
\hline 6 & Range $A_{A P}$ & 0.172 & 0.946 & -0.100 & 0.372 & 0.798 & 0.348 \\
\hline 7 & $\mathrm{D}_{\mathrm{ML}_{\mathrm{av}}}$ & 0.459 & 0.693 & -0.050 & 0.739 & -0.224 & 0.476 \\
\hline 8 & $\mathrm{D}_{\mathrm{AP}_{\mathrm{av}}}$ & -0.163 & -0.050 & 0.956 & 0.071 & 0.317 & 0.897 \\
\hline 9 & $\mathrm{~V}_{\mathrm{ML}_{\mathrm{av}}}$ & 0.946 & 0.258 & -0.163 & 0.938 & 0.238 & 0.043 \\
\hline 10 & $\mathrm{~V}_{\mathrm{AP}_{\mathrm{av}}}$ & 0.609 & 0.733 & -0.070 & 0.620 & 0.419 & 0.596 \\
\hline 11 & $A_{M_{a v}}$ & 0.921 & 0.336 & -0.169 & 0.946 & 0.215 & 0.126 \\
\hline 12 & $\mathrm{~A}_{\mathrm{AP}}{ }_{\mathrm{av}}$ & 0.461 & 0.866 & -0.056 & 0.657 & 0.274 & 0.573 \\
\hline 13 & $\mathrm{D}_{\mathrm{ML}_{\mathrm{RMS}}}$ & 0.491 & 0.689 & -0.047 & 0.767 & -0.166 & 0.466 \\
\hline 14 & $\mathrm{D}_{\mathrm{AP} R \mathrm{RMS}}$ & -0.180 & -0.081 & .0962 & 0.060 & 0.358 & 0.890 \\
\hline 15 & $\mathrm{~V}_{\text {MLRMS }}$ & 0.941 & 0.278 & -0.165 & 0.918 & 0.346 & 0.027 \\
\hline 16 & $\mathrm{~V}_{\mathrm{AP}_{\mathrm{RMS}}}$ & 0.586 & 0.757 & -0.088 & 0.561 & 0.576 & 0.533 \\
\hline 17 & $A_{M L_{R M S}}$ & 0.911 & 0.363 & -0.169 & 0.933 & 0.296 & 0.121 \\
\hline 18 & $\mathrm{~A}_{\mathrm{AP}}{ }_{\mathrm{RMS}}$ & 0.449 & 0.876 & -0.071 & 0.628 & 0.374 & 0.564 \\
\hline
\end{tabular}


The first principal component (PC1) was correlated with 13 variables in both the ML and AP directions with 0.4 used as the threshold for minimal significance of correlation. Due to their high correlation coefficients, the first principal component is a representative of mostly variations in the ML direction, i.e. the range of the displacement (Range $\left.D_{\mathrm{ML}}\right)$, velocity (Range $V_{\mathrm{ML}}$ ), and acceleration (Range $A_{\mathrm{ML}}$ ), the average velocity $\left(\mathrm{V}_{\mathrm{MLav}}\right)$ and acceleration $\left(A_{M L_{a v}}\right)$, the root mean square (RMS) velocity $\left(\mathrm{V}_{\mathrm{ML}} \mathrm{L}_{\mathrm{RS}}\right)$ and acceleration $\left(\mathrm{A}_{\mathrm{ML}} \mathrm{RMS}\right)$.

The second principal component (PC2) correlated with nine variables in both the ML and AP directions, i.e. the range of displacement in the ML direction (Range $D_{\mathrm{ML}}$ ), the range of the velocity in the AP direction (Range $V_{\mathrm{AP}}$ ), the range of the acceleration in the $\mathrm{ML}$ and $\mathrm{AP}$ direction (Range $A_{\mathrm{ML}}$ and Range $A_{\mathrm{AP}}$ ), the average displacement in the ML direction $\left(D_{M L_{a v}}\right)$, the average velocity and acceleration in the AP direction $\left(\mathrm{V}_{\mathrm{ML}_{\mathrm{av}}}\right.$ and $\left.\mathrm{V}_{\mathrm{AP}_{\mathrm{av}}}\right)$, the RMS displacement in the ML direction $\left(\mathrm{D}_{\mathrm{ML}_{\mathrm{RMS}}}\right)$, the RMS velocity and acceleration in the AP direction $\left(\mathrm{V}_{\mathrm{AP}_{\mathrm{RMS}}}\right.$ and $\left.\mathrm{A}_{\mathrm{AP} \mathrm{RMS}_{\mathrm{R}}}\right)$. Since $\mathrm{PC} 2$ correlated most strongly with the variables in the AP direction, it is a representative of the variation in the AP direction. Similarly, the third principal component (PC3) measured the variation in the AP direction due to same reasons as with the other principal components.

Since condition one of the MCTSIB comprised of all balance related sensory systems, it can be referred to as the reference and may represent the least extent of sway. The observation of a difference between the variables' correlations with the components across conditions indicated a difference in their underlying structure. One component may be sufficient for analysis since the variables were rotated. Since the first principal component represents the highest proportion of variance, analysis can be based on comparison of the loadings of this component in other conditions. Thus, the correlation of these variables in the ML direction is of importance for the purpose of analysis. Alternatively, analysis based on the variables that do not correlate strongly with PC1 in this case the AP directions can also be used. Their observations in the other conditions of the mCTSIB thus serve as an approach of analysis. The variables whose correlation coefficients appeared similar (with respect to $\mathrm{PC1}$, range of -0.114 to -0.180 for the Range $\mathrm{D}_{\mathrm{AP}}, \mathrm{D}_{\mathrm{AP}}$ av , and $\mathrm{D}_{\mathrm{AP}} \mathrm{RMS}$, range of 0.172 to 0.175 for Range $A_{A P}$ and RangeV $V_{A P}$, range of 0.461 to 0.609 for $\mathrm{A}_{\mathrm{AP} a v}$ and $\mathrm{V}_{\mathrm{AP} a v}$, range of 0.459 to 0.491 for $\mathrm{D}_{\mathrm{MLRMS}_{\mathrm{R}}}$ and $\mathrm{D}_{\mathrm{ML} a v}$, range of 0.449 to 0.586 for $\mathrm{V}_{\mathrm{AP} \text { RMS }}$ and $\mathrm{V}_{\mathrm{AP} \text { RMS }}$, range of 0.911 to 0.946 for $V_{M L_{a v}}, A_{M L_{a v}}, V_{M L_{R M S}}$, and $A_{M L_{R M S}}$, range of 0.774 to 0.843 for Range $\mathrm{D}_{\mathrm{ML}}$, Range $\mathrm{V}_{\mathrm{ML}}$, and Range $\mathrm{A}_{\mathrm{ML}}$ ) among subjects showed less variations in their standardised equivalents and as such had closely related correlations.

In mCTSIB's second condition, the additional variables that showed significant loadings for PC1 included: average displacement in the ML direction $\left(\mathrm{D}_{\mathrm{ML}_{\mathrm{av}}}\right)$, average acceleration in the $\mathrm{AP}$ direction $\left(\mathrm{A}_{\mathrm{AP}} \mathrm{av}\right)$, root mean square displacement in the $\mathrm{ML}$ direction $\left(\mathrm{D}_{\mathrm{ML}} \mathrm{RMS}_{\mathrm{S}}\right)$ and root mean square acceleration $\left(\mathrm{A}_{\mathrm{AP}} \mathrm{RMS}_{\mathrm{S}}\right)$ in the AP direction. While in $\mathrm{PC} 2$, the range of displacement in the AP direction (Range $\left.D_{\mathrm{AP}}\right)$, and the range of velocity in the ML direction (Range $V_{\mathrm{ML}}$ ) loaded with the component. In PC3, average velocity and acceleration in the $\mathrm{AP}$ direction $\left(\mathrm{V}_{\mathrm{AP}_{\mathrm{av}}}\right.$ and $\mathrm{A}_{\mathrm{AP}}$ av $)$ and the RMS of acceleration $\left(\mathrm{A}_{\mathrm{AP}} \mathrm{RMS}_{\mathrm{S}}\right)$ loaded with the principal component. Therefore, the first principal component described the sway in the ML direction, while the second and third were defined by the AP direction, based on the strongest correlations. More changes in the loadings of a number of variables were observed mainly in the AP direction in the first and third principal components in comparison of conditions one and two. This indicated the effectiveness of the AP direction to capture the sensory information of the visual system and highlighted an underlying difference in structure. However, there existed a close relationship with condition one as there were less changes in the correlations/loadings. This also indicated that the conditions where the subjects were standing on a firm surface, were closely related, i.e. conditions one and two. The component matrix is indicative of the underlying structure of the variables of the data set. However, variables may have similar correlation coefficients in the component matrix but vary in their magnitudes. As such, there was a need for further investigation into the actual variables. For simplicity, the RMS velocity was used for further analysis based on its relationship 
between the variables i.e. its strong correlations with other variables in both the ML and AP direction. The Bland and Altman plots for comparing the similarities between the ML and AP RMS velocities of conditions one and two are shown in Figure 6. The differences between the two are shown in the figure's vertical axis while its horizontal axis represents their respective means. The greater the mean difference between the two variables (indicated with the blue line) from the zero line (indicated with the thin dash lines), the greater their differences. The mean difference of the ML direction was smaller than that of the AP direction highlighting a greater amount of similarity in the ML direction between the two conditions. This also indicated that the AP direction was more differentiable and may be more sensitive to the absence or presence of the information from the visual sensory system. No consistent bias was observed in the ML direction between the two conditions as compared to that of the AP direction as the points were more evenly distributed above and below the zero line, indicating less certainty that the sway from condition two is more than those from condition one. In contrast to the ML direction, there is an increase in the consistency of the bias below the zero line in the AP direction indicating greater certainty that the amount of sway in condition two is more than that obtained from condition one. The negative value of the mean difference indicated that in general, the sway of condition one was less than condition two by its value $(0.09 \mathrm{~cm} / \mathrm{s}$ for the ML direction and $0.42 \mathrm{~cm} / \mathrm{s}$ for the AP direction). As indicated by the range of the limits of agreement, more variations were observed in the ML direction $(3.80 \mathrm{~cm} / \mathrm{s})$ as compared to that of the AP direction $(2.75 \mathrm{~cm} / \mathrm{s})$, an indication of less agreement in the ML direction of the two conditions. In comparison of the ratio of their means, the AP direction was approximately 4.67 times greater than that of the ML direction indicating more sway occurred in the AP direction as compared to the ML direction.

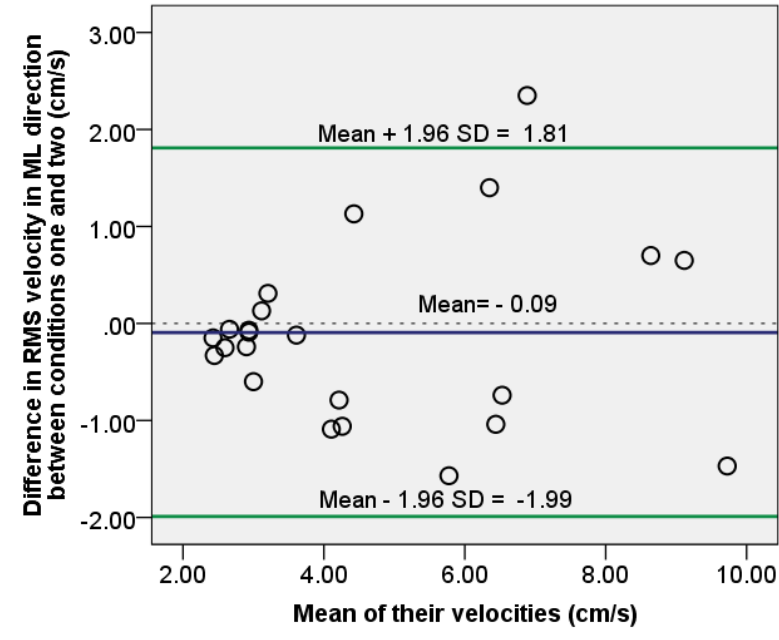

(a)



(b)

Figure 6. Bland and Altman plot of the correlations of variables with their components for (a) condition one (GEO) and (b) condition two (GEC) 
5.2. Comparison between conditions one and three
The rotated component matrix for mCTSIB's conditions 1 and 3 is shown in Table 2.

Table 2. Rotated component matrix for conditions one and three.

\begin{tabular}{|c|c|c|c|c|c|c|c|}
\hline \multicolumn{8}{|c|}{ mCTSIB } \\
\hline \multirow[b]{2}{*}{ Number } & \multirow[b]{2}{*}{ Variables } & \multicolumn{3}{|c|}{ Condition 1} & \multicolumn{3}{|c|}{ Condition 3} \\
\hline & & PC1 & PC2 & PC3 & PC1 & PC2 & PC3 \\
\hline 1 & RangeD $_{\mathrm{ML}}$ & 0.780 & 0.503 & -0.064 & 0.568 & 0.220 & -0.002 \\
\hline 2 & RangeD $_{\mathrm{AP}}$ & -0.114 & -0.081 & 0.857 & 0.396 & 0.173 & 0.834 \\
\hline 3 & RangeV $_{\mathrm{ML}}$ & 0.843 & 0.399 & -0.222 & 0.694 & 0.669 & 0.065 \\
\hline 4 & RangeV $_{\mathrm{AP}}$ & 0.175 & 0.934 & -0.096 & 0.284 & 0.880 & 0.230 \\
\hline 5 & RangeA $_{\mathrm{ML}}$ & 0.774 & 0.541 & -0.199 & 0.634 & 0.719 & 0.099 \\
\hline 6 & Range $_{\mathrm{AP}}$ & 0.172 & 0.946 & -0.100 & 0.331 & 0.885 & 0.203 \\
\hline 7 & $\mathrm{D}_{\mathrm{ML}_{\mathrm{av}}}$ & 0.459 & 0.693 & -0.050 & 0.049 & 0.171 & 0.167 \\
\hline 8 & $\mathrm{D}_{\mathrm{AP}}{ }_{\mathrm{av}}$ & -0.163 & -0.050 & .0956 & -0.117 & 0.143 & 0.964 \\
\hline 9 & $\mathrm{~V}_{\mathrm{ML}_{\mathrm{av}}}$ & 0.946 & 0.258 & -0.163 & 0.955 & 0.208 & -0.071 \\
\hline 10 & $\mathrm{~V}_{\mathrm{AP}_{\mathrm{av}}}$ & 0.609 & 0.733 & -0.070 & 0.960 & 0.139 & 0.123 \\
\hline 11 & $A_{M_{a v}}$ & 0.921 & 0.336 & -0.169 & 0.963 & 0.205 & -0.078 \\
\hline 12 & $\mathrm{~A}_{\mathrm{AP}} \mathrm{av}$ & 0.461 & 0.866 & -0.056 & 0.965 & 0.116 & 0.096 \\
\hline 13 & $\mathrm{D}_{\mathrm{ML}_{\mathrm{RMS}}}$ & 0.491 & 0.689 & -0.047 & 0.097 & 0.156 & 0.158 \\
\hline 14 & $\mathrm{D}_{\text {APRMS }}$ & -0.180 & -0.081 & 0.962 & -0.085 & 0.099 & 0.978 \\
\hline 15 & $\mathrm{~V}_{\mathrm{ML}_{\mathrm{RMS}}}$ & 0.941 & 0.278 & -0.165 & 0.939 & 0.266 & -0.055 \\
\hline 16 & $\mathrm{~V}_{\mathrm{AP}} \mathrm{RMS}$ & 0.586 & 0.757 & -0.088 & 0.857 & 0.416 & 0.180 \\
\hline 17 & $A_{M L R M S}$ & 0.911 & 0.363 & -0.169 & 0.942 & 0.292 & -0.055 \\
\hline 18 & $\mathrm{~A}_{\mathrm{AP}} \mathrm{RMS}_{\mathrm{S}}$ & 0.449 & 0.876 & -0.071 & 0.881 & 0.388 & 0.149 \\
\hline
\end{tabular}


In condition three, similar correlated variables were observed as in mCTSIB's conditions one and two above. However, there were stronger correlations in the AP direction. This is an indication of a change in variation of sway in that direction as compared to condition one. Relatively smaller changes in correlation were observed in the ML direction in comparison with that of the AP direction. The first principal component (PC1) was strongly correlated with both the ML and AP directions. Strongly correlated variables were due to their pattern of variation rather than the magnitudes as the variables were all standardised. The second and third principal component (PC2 and $\mathrm{PC} 3$ ) correlated strongly with the AP direction suggesting similarities to condition one. Since PC1 represented most of the variation (60.87\%) in the data set, its comparison across both conditions was important. Comparing its ML and AP correlations, a greater variation occurred in the AP direction than that of ML direction and as such the AP direction may be more sensitive to the absence or presence of sensory input information. The Bland and Altman plot for comparing the similarities of the ML and AP RMS velocities between conditions one and three are shown in Figure 7. The differences between the two corresponding variables are shown in the vertical axis while the horizontal axis represent their respective means. The mean difference $(0.03 \mathrm{~cm} / \mathrm{s})$ of the ML direction was smaller than that of the AP direction $(0.85 \mathrm{~cm} / \mathrm{s})$ and thus indicating greater similarity in the ML

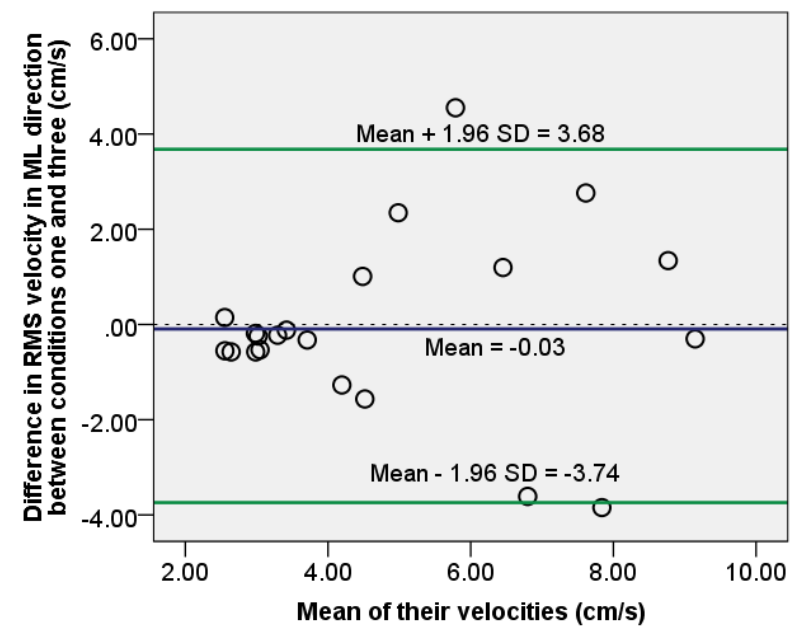

(a) direction between the two conditions. A smaller consistent bias was observed in the ML direction between the conditions given that the points were more evenly distributed above and below the zero line (indicated with the thin dash lines). This highlighted a reduced certainty that the sway in condition three was greater than condition one and vice-versa. In contrast to the ML direction, the AP direction provided a greater consistency of bias towards condition three, indicating that the sway in condition three was greater than the sway in condition one in magnitude. As indicated by the range of the limits of agreement, there existed a larger variation in the ML direction $(7.42 \mathrm{~cm} / \mathrm{s})$ as compared to that of the AP direction (3.91 $\mathrm{cm} / \mathrm{s}$ ). Comparing the ratio of their means, the AP direction was approximately 28.33 times more than that of the ML direction. This indicated a greater sway occurs in the AP direction as compared to the ML direction. Based on the comparisons of the mean differences between the AP directions of conditions one and two $(0.42 \mathrm{~cm} / \mathrm{s})$, and conditions one and three $(0.85 \mathrm{~cm} / \mathrm{s})$, it was noted that the vestibular system interacted more effectively using the somatosensory system as compared to its interaction with the visual system in maintaining standstill balance. The somatosensory system has more coherence across the subjects as compared to the visual system as indicated by the range of the limits of agreement between conditions two $(2.75 \mathrm{~cm} / \mathrm{s})$ and three $(3.91 \mathrm{~cm} / \mathrm{s})$ in respect to condition one.

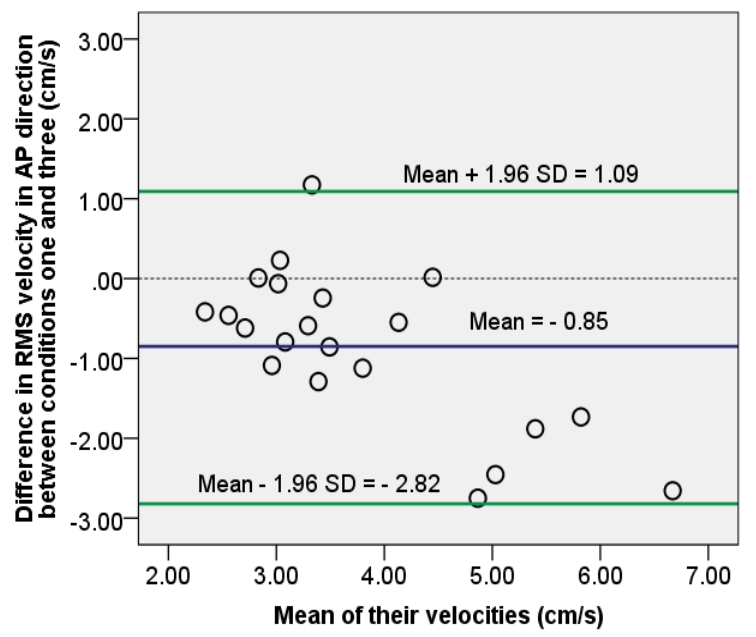

(b)

Figure 7. Bland and Altman plots of the correlations of variables with their components for (a) condition one (GEO) and (b) condition three (FEO) 


\subsection{Comparison between conditions one and four}

The rotated component matrix for mCTSIB's conditions one and four is shown in Table 3.

Table 3. Rotated component matrix for mCTSIB's conditions one and four.

\begin{tabular}{|c|c|c|c|c|c|c|c|}
\hline \multicolumn{8}{|c|}{ mCTSIB } \\
\hline \multirow[b]{2}{*}{ Number } & \multirow[b]{2}{*}{ Variables } & \multicolumn{3}{|c|}{ Condition 1} & \multicolumn{3}{|c|}{ Condition 4} \\
\hline & & PC1 & PC2 & PC3 & PC1 & PC2 & PC3 \\
\hline 1 & RangeD $_{M L}$ & 0.780 & 0.503 & -0.064 & 0.657 & 0.627 & 0.145 \\
\hline 2 & RangeD $_{\mathrm{AP}}$ & -0.114 & -0.081 & 0.857 & 0.484 & 0.356 & 0.582 \\
\hline 3 & RangeV $_{M L}$ & 0.843 & 0.399 & -0.222 & 0.850 & 0.294 & 0.275 \\
\hline 4 & RangeV $_{\mathrm{AP}}$ & 0.175 & 0.934 & -0.096 & 0.825 & 0.216 & 0.232 \\
\hline 5 & Range $A_{M L}$ & 0.774 & 0.541 & -0.199 & 0.880 & 0.260 & 0.190 \\
\hline 6 & RangeA $A_{A P}$ & 0.172 & 0.946 & -0.100 & 0.838 & 0.153 & 0.166 \\
\hline 7 & $\mathrm{D}_{\mathrm{ML}_{\mathrm{av}}}$ & 0.459 & 0.693 & -0.050 & 0.143 & 0.977 & 0.051 \\
\hline 8 & $\mathrm{D}_{\mathrm{AP}}$ & -0.163 & -0.050 & 0.956 & 0.123 & 0.018 & 0.972 \\
\hline 9 & $\mathrm{~V}_{\mathrm{ML}_{\mathrm{av}}}$ & 0.946 & 0.258 & -0.163 & 0.934 & 0.262 & 0.141 \\
\hline 10 & $\mathrm{~V}_{\mathrm{AP}} \mathrm{av}$ & 0.609 & 0.733 & -0.070 & 0.950 & 0.104 & 0.106 \\
\hline 11 & $A_{M L}$ & 0.921 & 0.336 & -0.169 & 0.946 & 0.194 & 0.139 \\
\hline 12 & $\mathrm{~A}_{\mathrm{AP}} \mathrm{av}$ & 0.461 & 0.866 & -0.056 & 0.976 & 0.054 & 0.057 \\
\hline 13 & $\mathrm{D}_{\mathrm{ML}_{\mathrm{RM}}}$ & 0.491 & 0.689 & -0.047 & 0.191 & 0.972 & 0.068 \\
\hline 14 & $\mathrm{D}_{\mathrm{AP}_{\mathrm{RMS}}}$ & -0.180 & -0.081 & 0.962 & 0.146 & 0.048 & 0.981 \\
\hline 15 & $\mathrm{~V}_{\mathrm{ML}}$ & 0.941 & 0.278 & -0.165 & 0.923 & 0.269 & 0.173 \\
\hline 16 & $\mathrm{~V}_{\mathrm{AP}} \mathrm{RMS}$ & 0.586 & 0.757 & -0.088 & 0.951 & 0.125 & 0.153 \\
\hline 17 & $A_{M_{R M S}}$ & 0.911 & 0.363 & -0.169 & 0.939 & 0.196 & 0.152 \\
\hline 18 & $\mathrm{~A}_{\mathrm{AP}} \mathrm{RMS}$ & 0.449 & 0.876 & -0.071 & 0.977 & 0.072 & 0.085 \\
\hline
\end{tabular}


Condition four which is defined by only the vestibular system showed more difference in structure (indicated by the correlation coefficients of the variables that loaded with the components) from that of condition one as compared to conditions two and three. The first principal component also indicated similar changes in the correlations of the variables in the ML direction indicating that the ML direction may be less sensitive to the contribution of the vestibular sensory system.

The Bland and Altman plots for comparing the similarities or dissimilarities of the ML and AP RMS velocities between condition one and four are shown in Figure 8. The mean difference (0.46 $\mathrm{cm} / \mathrm{s}$ ) of the ML direction appeared smaller than that of the AP direction $(1.93 \mathrm{~cm} / \mathrm{s})$ and highlighting a greater similarity in the ML direction between the two conditions. This also indicated that the AP direction was more sensitive to the possible presence of the information from the vestibular system. A

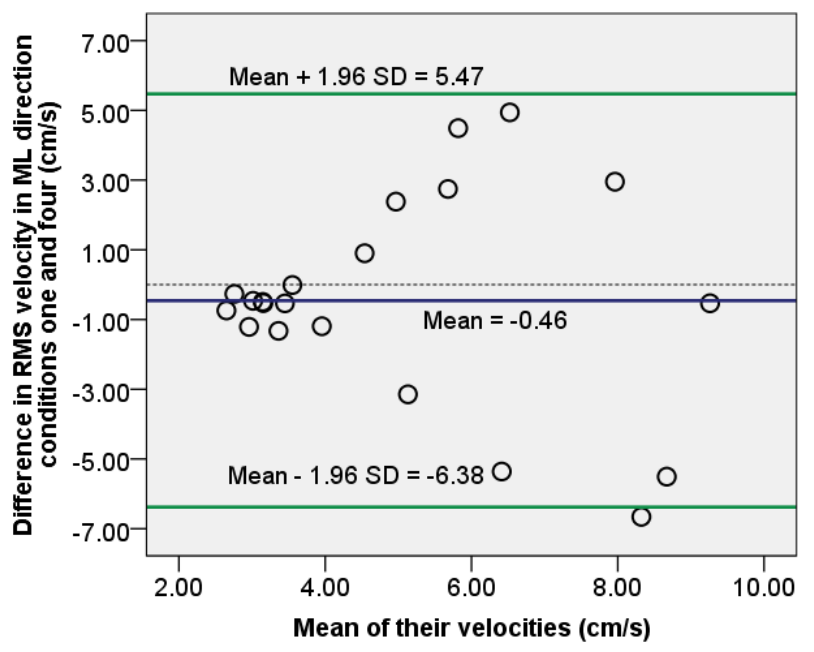

(a) smaller consistent bias was observed in the ML direction between the conditions, as the points were more evenly distributed above and below the zero line (indicated with the thin dash lines), highlighting less certainty that the sway in condition four was greater than that of condition one or vice-versa. In contrast to the ML direction, there was more consistency of bias towards the AP direction, indicating that the sway of condition four was more than that of condition one. As indicated by the range of the limits of agreement, there existed a larger variation in the ML direction $(11.85 \mathrm{~cm} / \mathrm{s})$ as compared to that of the AP direction $(7.17 \mathrm{~cm} / \mathrm{s})$. In comparison of the ratio of their means, the AP direction was approximately 4.20 times larger than that of the ML direction-suggesting more sway occurred in the AP direction as compared to the ML direction. The vestibular system in comparison with the somatosensory and visual systems showed smaller coherence of sway across the subjects as indicated by the range of the limits of agreement $(7.17 \mathrm{~cm} / \mathrm{s})$.



(b)

Figure 8. Bland and Altman plot of the correlations of variables with their components for (a) condition one (GEO) and (b) condition four (FEC) 
The median and interquartile ranges of the RMS velocity for the four conditions of the MCTSIB are shown in Table 4. Considering the AP direction, the mean and the variance values are in ascending order of magnitude from conditions one to four. The interquartile range (IQR) indicated a closely related characteristics of sway were exhibited by subjects in condition one as compared to the other conditions. Also, condition two had less variation as compared to condition three, with condition four having the greatest variation. The results obtained from the median based Levene's test for the conditions showed homogeneity of variance across the ML direction $(\mathrm{F}(3,80)=0.066, p=0.978)$ while the AP direction showed heterogeneity $(\mathrm{F}(3,80)=3.61, p=0.017)$. The Friedman test for significant differences showed no statistically significant difference across the ML condition $\left(x^{2}(3)=6.52, p>0.05\right) \quad$ while there was significant difference across the AP condition $\left(x^{2}(3)=32.71, p<0.01\right)$. This highlighted that the AP direction was more sensitive to the balance related sensory information as compared to the ML direction. Post hoc analysis with the Sign test was conducted between the paired conditions in the AP direction (i.e. conditions 1 and 2, 1 and 3, 1 and 4, 2 and 3,2 and 4, 3 and 4) with a Bonferroni correction resulting in the significance level set at $\mathrm{p}<0.0083\left(\alpha=\frac{0.05}{6}=0.0083\right)$. The median (IQR) for the conditions in the AP direction were 3.15 (2.71 to 3.89 ), 3.50 (2.99 to 4.73 ), 3.59 (2.97 to 5.34) and 4.24 (3.62 to 7.55) respectively. There was no significant difference between conditions one and two $(p=0.027)$, and conditions two and three $(p=0.383)$, while there were significant differences between other comparisons, i.e. one and three $(p=0.007)$, one and four $(p<0.001)$, two and four $(p<0.001)$ and three and four $(p=$ $0.001)$. This is an indication that condition two (vestibular and the somatosensory system) was more similar to condition one (vestibular, somatosensory and visual system) as compared to condition three (vestibular system and visual system) and four (vestibular system). In summary, the study indicated that:

i. The vestibular system performed better with the somatosensory system than the visual system in maintaining balance in a standstill position. The finding in this study is in agreement with those obtained in [23]. The findings in [23] suggested that healthy adults were more dependent on their somatosensory system as compared to their visual system in a well-lit environment [23] [24].

ii. The AP direction was more sensitive to balance related sensory information than their ML counterparts in a standstill position. The finding in this study is in agreement with those obtained in [25]. In their study, they observed that the average centre of pressure (COP) velocity and the RMS of COP in the AP direction was capable of differentiating between patients with chronic lower back pain (CLBP) and healthy subjects. However, no significant difference was observed in the ML direction between the two groups using same measures [25].

iii. There was less variability from the somatosensory system across individuals as compared to the vestibular and visual system when considering balance in a standstill position. The findings in this study agreed with similar findings in [26]. In their study, they observed that the somatosensory system had less intersubject variability as compared to the visual and vestibular system in an upright posture using the ankle strategy [26].

Table 4. The median and interquartile range (IQR) of the RMS velocity in ML and AP conditions of the $\mathrm{mCTSIB}$.

\begin{tabular}{|c|c|c|c|c|}
\hline \multirow[t]{2}{*}{$\begin{array}{l}\text { Conditions of the modified clinical test for sensory } \\
\text { interaction with balance (mCTSIB) }\end{array}$} & \multicolumn{2}{|c|}{$\begin{array}{l}\text { RMS velocity in } \\
\text { the ML } \\
\text { direction }\end{array}$} & \multicolumn{2}{|c|}{$\begin{array}{l}\text { RMS velocity in } \\
\text { the AP direction }\end{array}$} \\
\hline & Median & IQR & Median & IQR \\
\hline Condition one (all balance-related sensory systems) & 3.56 & 3.77 & 3.15 & 1.18 \\
\hline Condition two (somatosensory and vestibular systems) & 3.86 & 3.73 & 3.50 & 1.74 \\
\hline $\begin{array}{l}\text { Condition three (visual and somatosensory and } \\
\text { vestibular systems) }\end{array}$ & 3.81 & 2.84 & 3.59 & 2.37 \\
\hline $\begin{array}{l}\text { Condition four (visual and somatosensory and } \\
\text { vestibular systems) }\end{array}$ & 4.02 & 3.11 & 4.24 & 3.93 \\
\hline
\end{tabular}




\section{Conclusion}

Principal component analysis (PCA) was used to investigate accelerometry obtained balanced related variables in twenty-one healthy adult subjects using the Modified Clinical Test of Sensory Interaction on Balance (mCTSIB). The analysis was carried out on the mediallateral (ML) and anterior-posterior (AP) directions. Firstly, it was observed that the AP direction was more sensitive to balance related sensory information as compared to the ML direction. Secondly, more variance in sway was observed in the visual and vestibular sensory systems as compared to the somatosensory systems. Finally, the vestibular system performed more effectively with the somatosensory system than the visual system in maintaining balance. The findings of the study could assist in better interpreting accelerometry recorded data obtained through mCTSIB for diagnosing balance dysfunctions.

\section{References}

1. Pasma, J., Engelhart, D., Schouten, A., van der Kooij, H., Maier, A. and Meskers, C. ( 2014) Impaired standing balance: The clinical need for closing the loop. Neuroscience, 267, pp.157165.

2. Duysens, J., Clarac, F. and Cruse, H. (2000) Load-regulating mechanisms in gait and posture: comparative aspects. Physiological Reviews, 80(1), pp.83-133.

3. Shumway-Cook, A., Horak, F. (1986) Assessing the influence of sensory interaction on balance. Phys. Ther., 66, pp.1548-1550.

4. Cohen, H.; Blatchly, C.A., Gombash, L.L. (1993) A study of clinical test of sensory interaction and balance. Phys. Ther., 73, pp.346-351.

5. Chen, T., Xu, G., Zhou, G., Wang, J., Chan, P. and Du, Y. (2014) Postural sway in idiopathic rapid eye movement sleep behavior disorder: A potential marker of prodromal Parkinson's disease. Brain Research, 1559, pp.26-32.
6. Mancini, M., Horak, F., Zampieri, C., Carlson-Kuhta, P., Nutt, J. and Chiari, L. (2011) Trunk accelerometry reveals postural instability in untreated Parkinson's disease. Parkinsonism \& Related Disorders, 17(7), pp.557-562.

7. Cao, L., Chua, K., Chong, W., Lee, H. and $\mathrm{Gu}, \mathrm{Q}$. (2003) A comparison of PCA, KPCA and ICA for dimensionality reduction in support vector machine. Neurocomputing, 55(1-2), pp.321-336.

8. Promsri, A. and Federolf, P. (2020) Analysis of postural control using principal component analysis: The relevance of postural accelerations and of their frequency dependency for selecting the number of movement components. Frontiers in Bioengineering and Biotechnology, 8:480. doi: 10.3389/fbioe.2020.00480

9. Labbe, D., de Guise, J., Mezghani, N., Godbout, V., Grimard, G., Baillargeon, D., Lavigne, P., Fernandes, J., Ranger, P. and Hagemeister, N. (2010) Feature selection using a principal component analysis of the kinematics of the pivot shift phenomenon. Journal of Biomechanics, 43(16), pp.3080-3084.

10. Haid, T., Doix, A., Nigg, B. and Federolf, P. (2018) Age effects in postural control analyzed via a principal component analysis of kinematic data and interpreted in relation to predictions of the optimal feedback control theory. Frontiers in Aging Neuroscience, 10:22. doi: 10.3389/fnagi.2018.00022.

11. Brodie, M., Menz, H. and Lord, S. (2013) Age-associated changes in head jerk while walking reveal altered dynamic stability in older people. Experimental Brain Research, 232(1), pp.51-60.

12. Winter, D.; Patla, A.; Prince, F.; Ishac, M.; Gielo-Perczak, K. (1998) Stiffness control of balance in quiet standing. $J$. Neurophysiol., 80, pp.1211-1221. 
13. Mayagoitia, R.; Lötters, J.; Veltink, P.; Hermens, H. (2002) Standing balance evaluation using a triaxial accelerometer. Gait Posture, 16, pp.55-59.

14. Ojie, O., Saatchi, R. and Saatchi, M. (2020) Demonstration of the effect of centre of mass height on postural sway using accelerometry for balance analysis. Technologies, 8(2), p.20.

15. Jolliffe, I. and Cadima, J. (2016) Principal component analysis: a review and recent developments. Philosophical

Transactions of the Royal Society A 374: 20150202.

16. Rencher, A.C. (2002) Methods of multivariate analysis. Wiley.

17. Cattell, R. and Vogelmann, S. (1977) A Comprehensive trial of the scree and $\mathrm{Kg}$ criteria for determining the number of factors. Multivariate Behavioral Research, 12(3), pp.289325.

18. Kaiser, H. (1958) The varimax criterion for analytic rotation in factor analysis. Psychometrika, 23(3), pp.187-200.

19. Single, R. (1996) Multivariate data analysis with readings. The American Mathematical Monthly, Washington, 103(5), pp.444.

20. Ho, R. (2006) Handbook of univariate and multivariate data analysis and interpretation with spss. Boca Raton: Chapman \& Hall/CRC.

21. OJie, O. D. and Saatchi, R. (2020) Development and evaluation of an accelerometry system based on inverted pendulum to measure and analyze human balance. In: BALL, Andrew, GELMAN, Len and RAO, B.K.N., (eds.) Advances in Asset Management and Condition Monitoring: COMADEM 2019. Smart Innovation, Systems and Technologies, 166 . Springer, 11291141.
22. Bland, J.M. and Altman, D. (1986) Statistical methods for assessing agreement between two methods of clinical measurement. The Lancet, 327(8476), pp.307-310.

23. Peterka, R. (2002) Sensorimotor integration in human postural control. Journal of Neurophysiology, 88(3), pp.1097-1118.

24. Horak, F. (2006) Postural orientation and equilibrium: what do we need to know about neural control of balance to prevent falls?. Age and Ageing, 35(suppl_2), pp.ii7-ii11.

25. della Volpe, R., Popa, T., Ginanneschi, F., Spidalieri, R., Mazzocchio, R. and Rossi, A. (2006) Changes in coordination of postural control during dynamic stance in chronic low back pain patients. Gait \& Posture, 24(3), pp.349-355.

26. Fukuoka, Y., Nagata, T., Ishida, A. and Minamitani, H. (2001) Characteristics of somatosensory feedback in postural control during standing. IEEE Transactions on Neural Systems and Rehabilitation Engineering, 9(2), pp.145-153.

\section{Creative Commons Attribution License 4.0 (Attribution 4.0 International, CC BY 4.0)}

This article is published under the terms of the Creative Commons Attribution License 4.0 https://creativecommons.org/licenses/by/4.0/deed.en_US 